\title{
Eosinophilic meningoencephalitis due to neurotoxocariasis presenting as gross motor developmental regression in an infant: A Case Report
}

\author{
TNP Suriapperuma, V Koculen, C Udagedara, KP Weerasekara \\ Sri Lankan Journal of Infectious Diseases 2021 Vol.11(2):107-111 \\ DOI: http://dx.doi.org/10.4038/sljid.v11i2.8342
}

\begin{abstract}
Eosinophilic meningitis is diagnosed when CSF contains $>10$ eosinophils $/ \mathrm{ml}$ or at least $10 \%$ of eosinophils as a proportion of CSF leucocytes. The most common etiology of eosinophilic meningitis is tissue migrating helminths. We report a ten month old infant with neurotoxocariasis presenting as gross motor developmental regression and features of meningoencephalitis which is very rare in this age group. A dramatic improvement was observed within a few days of starting Albendazole and prednisolone and the patient recovered without residual neurological deficit. We conclude that neurotoxocariasis should be suspected in the presence of neurological symptoms along with eosinophilia in blood and/or CSF.
\end{abstract}

Keywords: Eosinophilic meningoencephalitis, neurotoxocariasis, tissue migrating helminths, antitoxocara antibodies, anthelminthics

\section{Introduction}

Eosinophilia in cerebrospinal fluid (CSF) is rare. Eosinophilic meningitis is diagnosed when CSF contains $>10$ eosinophils $/ \mathrm{ml}$ or at least $10 \%$ of eosinophils as a proportion of CSF leucocytes. The most common etiology of eosinophilic meningitis is tissue migrating helminths. ${ }^{1}$ Here we report the youngest patient in the literature with eosinophilic meningoencephalitis due to neurotoxocariasis, which is very rare in any age group.

${ }^{1}$ Lady Ridgeway Hospital for Children, Sri Lanka

${ }^{2}$ Department of Paediatrics, Faculty of Medicine, University of Keleniya, Sri Lanka

Address for correspondence: Dr TNP Suriapperuma, Faculty of Medicine, University of Kelaniya, Talagolla Road, Ragama, Sri Lanka.. Telephone: +940715121047.E-mail: thari1701@gmail.com

(iD https://orcid.org/0000-0002-8129-1297

Received 28 December 2020 and revised version accepted 24 April 2021.

(c) (7) use, distribution, and reproduction in any medium, provided the original author and source are credited. 


\section{Case report}

A ten month old previously healthy girl presented with a history of intermittent low-grade fever for 12 days and a high-pitched cry with irritability for four days. There were no convulsions. She was developmentally age-appropriate in all domains. With this illness, she couldn't walk with support, weight bear, or maintain sitting balance. There was no regression in other domains. There was exposure to a pet dog at home. She was adequately grown with no recent growth faltering. Her weight was $8.7 \mathrm{~kg}$ (1SD to median), length was $0.72 \mathrm{~m}$ (1SD to median) and occipitofrontal circumference was $0.45 \mathrm{~m}$ (25th to 50th centiles). She was irritable, less active, and became more irritable to sound and light. Clinically there was a left-sided convergent squint. There was no organomegaly. Muscle tone, power, and reflexes were normal in upper and lower limbs. The rest of the examination was unremarkable.

Investigations revealed leukocytosis $(16,200 / \mu \mathrm{L})$ with eosinophilia (absolute eosinophil count $(\mathrm{AEC})-2198 / \mu \mathrm{L})$ and thrombocytosis $(642,000 / \mu \mathrm{L})$. Her eosinophil count was monitored, and AEC increased to $3852 / \mu \mathrm{L}, 4500 / \mu \mathrm{L}, 5200 / \mu \mathrm{L}$ and, $5400 / \mu \mathrm{L}$ over a period of one week. Her haemoglobin was $11.7 \mathrm{~g} / \mathrm{dl}$, erythrocyte sedimentation rate $32 \mathrm{~mm} / 1 \mathrm{st}$ hour and $\mathrm{C}$ reactive protein $25 \mathrm{mg} / \mathrm{dl}$. The blood picture showed severe eosinophilia with normal morphology in other cells.

Contrast-enhanced computerized tomography of the brain, magnetic resonant imaging, magnetic resonant arteriography, and venography of the brain were normal. CSF analysis revealed total cells of $438 / \mathrm{mm}^{3}$ of which $40 \%$ were eosinophils, $35 \%$ polymorphs and the rest were lymphocytes. CSF protein was $32 \mathrm{mg} / \mathrm{dl}$. CSF sugar was $2.4 \mathrm{mmol} / \mathrm{L}$ with blood sugar of 5.6 $\mathrm{mmol} / \mathrm{L}$. Bone marrow (BM) biopsy showed reactive changes with increased eosinophils and eosinophilic precursors, without evidence of malignancy. Ultrasound scan of abdomen and echocardiogram did not reveal evidence of organ infiltration.

Serum Toxocara IgG was positive with an absorbance reading of 0.7 OD units. It was analyzed using a commercially available enzyme-linked immunosorbent assay to detect Toxocara IgG (DRG, USA; cut off value 0.3 OD units). Toxoplasma IgM and IgG were negative. Blood, CSF, and BM cultures were sterile. The ophthalmologic assessment did not reveal evidence of ocular toxocariasis (OT).

She was commenced on broad-spectrum intravenous antibiotics as she had clinical features of meningoencephalitis to which she did not respond and eosinophilia gradually worsened. After confirming the diagnosis, she was started on oral albendazole $200 \mathrm{mg}$ twice daily with prednisolone $1 \mathrm{mg} / \mathrm{kg} /$ day for four weeks. The absolute eosinophil count came down to $300 / \mu \mathrm{L}$ within 72 hours of treatment and remained within the normal range thereafter. Her eye movements recovered fully and there was rapid improvement in gross motor development within two weeks.

The timeline of her clinical course is shown in Figure 1 


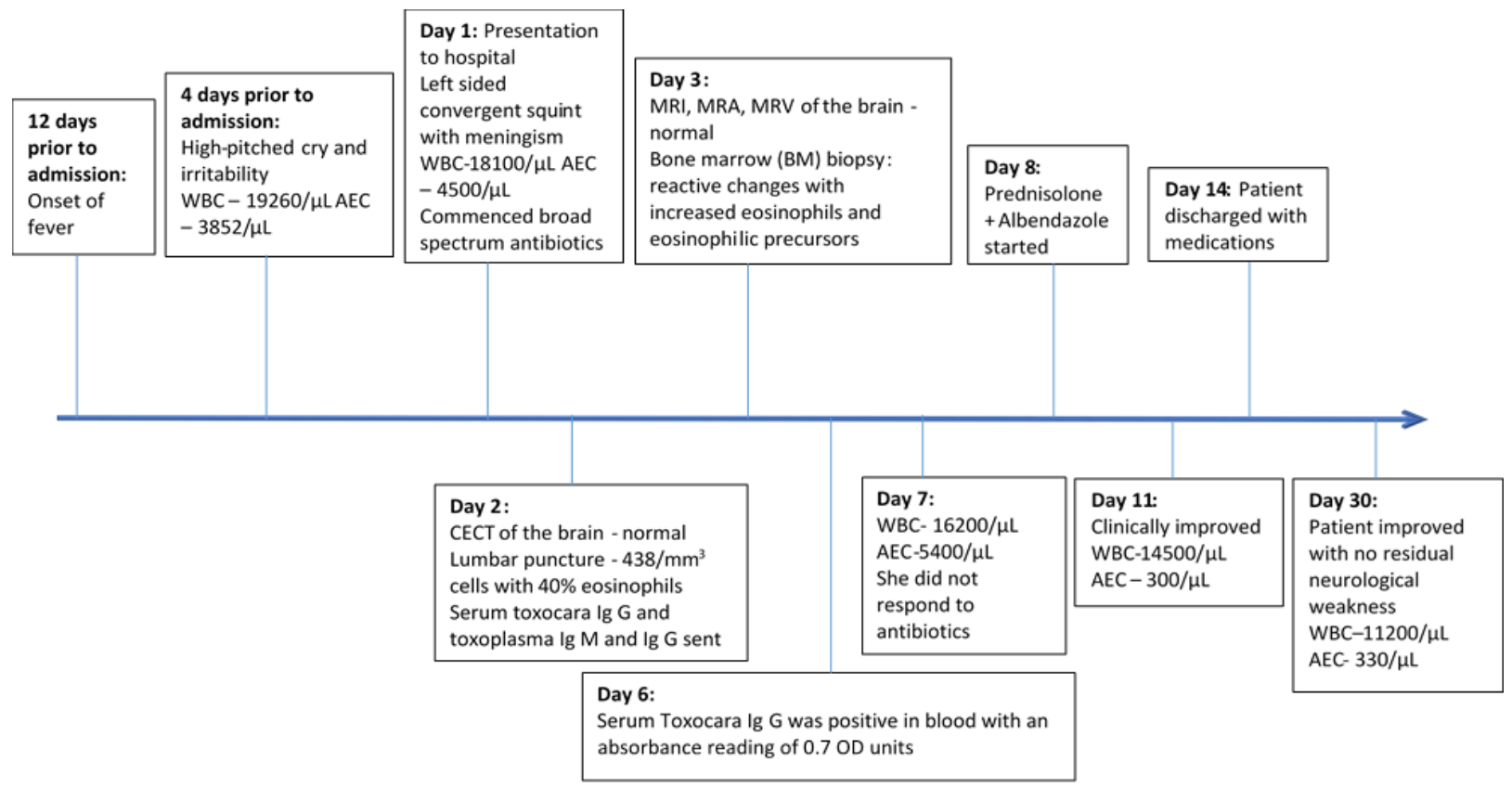

Figure 1: Timeline of the Patient's Clinical Course

\section{Discussion}

Toxocariasis is a zoonotic disease caused by dog and cat roundworms (Toxocara canis and Toxocara cati). There are four clinical forms of toxocariasis depending on organ involvement: visceral toxocariasis (VT), ocular toxocariasis (OT), covert or common toxocariasis, and neurotoxocariasis. ${ }^{2,3}$

An infected dog or cat sheds unembryonated eggs with feces which takes 2-4 weeks for embryonation in the environment. Unembryonated eggs are not infectious. When humans or other animals ingest embryonated eggs, they become infected. In the intestine, these eggs hatch, and the larvae migrate through the intestinal wall and enter various internal organs. Toxocara cannot complete the life cycle in the human body. Infected humans do not excrete Toxocara eggs and therefore do not transmit the disease. ${ }^{4,5}$

Toxocara larvae can cross the blood-brain-barrier and invade the brain and spinal cord causing neurotoxocariasis. ${ }^{5,6}$ Most individuals are asymptomatic. ${ }^{6}$ Acute disseminated encephalitis, meningoencephalitis, myelitis, central nervous system vasculitis, eosinophilic meningitis, cerebritis, epilepsy, and neuropsychological deficits are well recognized clinical manifestations with neurotoxocariasis. ${ }^{5,6}$ Neurotoxocariasis is diagnosed in the presence of a compatible clinical and radiological picture, a high titre of anti- Toxocara antibody in serum, and/or CSF, eosinophilia in the serum and/or CSF, and clinical and radiological improvement following anthelmintics and absence of alternative diagnoses. ${ }^{2,6}$ 
Our patient presented with an acute gross motor regression, left-sided convergent squint, clinically suspected meningoencephalitis, eosinophilia in serum, and CSF with low CSF sugar. In approximately half of the individuals with neurotoxocariasis, the CSF protein was not significantly elevated. ${ }^{2,7}$ In rare cases, low CSF sugar levels were reported. ${ }^{2}$ Her anti-Toxocara IgG antibodies in serum were positive and she showed dramatic improvement to specific treatment, on the basis of which eosinophilic meningoencephalitis due to neurotoxocariasis was diagnosed in our patient. However, we could not elicit any risk factors for toxocariasis in the history nor could we demonstrate CNS imaging abnormalities.

According to a systematic review, only $65.2 \%$ of patients diagnosed with neurotoxocariasis had abnormalities in the CT scan and $57.4 \%$ had abnormal findings in the MRI scan of the brain. ${ }^{2}$ As we have not performed MRI of the spine, we could not comment on myelitis.

Recommended treatment for VT is $400 \mathrm{mg}$ of oral albendazole or $100-200 \mathrm{mg}$ of mebendazole twice daily for 5 days. The same dose is used for adults and paediatric patients. ${ }^{4}$ OT always needs to be treated with an anthelminthic and steroids. Neurotoxocariasis needs treatment for a longer duration than VT and the use of steroids is controversial. ${ }^{5}$ Most of the reported cases of neurotoxocariasis were treated with an anthelminthic and steroids for nearly four weeks., Steroids are thought to reduce inflammation, immunologic manifestations, and to increase albendazole levels by $50 \%$ in serum. ${ }^{8}$

\section{Conclusion}

We suggest that neurotoxocariasis should be suspected in the presence of neurological symptoms along with eosinophilia in blood and/or CSF. Patient history, clinical presentation, positive investigation findings, and rapid response to treatment were in favour of neurotoxocariasis in our patient. Early clinical suspicion, prompt investigations, and treatment are required to minimize permanent neurological sequelae.

\section{Declarations}

Conflict of Interest statement - There are no conflicts of interest

Acknowledgments - We would like to acknowledge our patient and her family and all the ward staff who contributed in managing this patient.

Funding source - source(s) of support / funding - Self-funding

Ethics statement and consent for publication - Verbal consent was taken from the parents for publication

Author contributions - TNPS was the main contributor of writing the case report. VK and CU contributed to writing the discussion. KPW

supervised and edited the final case report.

\section{References}

1. Graeff-Teixeira C, da Silva ACA, Yoshimura K. Update on eosinophilic meningoencephalitis and its clinical relevance. Clinical Microbiology Reviews 2009; 22(2):322-48.

doi: 10.1128/CMR.00044-08

2. Deshayes S, Bonhomme J, Blanchardiere AL. Neurotoxocariasis: a systematic literature review. Infection 2016; 44:565-574. doi 10.1007/s15010-016-0889-8

3. Chen J, Liu Q, Liu G, et al. Toxocariasis: a silent threat with a progressive public health impact. Infectious Diseases of Poverty 2018; 7:59. doi.org/10.1186/s40249-018-0437-0. 
4. Woodhall DM, Fiore AE. Toxocariasis: A review for pediatricians. Journal of the Pediatric Infectious Diseases Society 2014; 3(2):154-59. doi:10.1093/jpids/pit066

5. Fan $\mathrm{C}$, Holland $\mathrm{CV}$, Loxton $\mathrm{K}$, et al. Cerebral toxocariasis: Silent progression to neurodegenerative disorder? Clinical Microbiology reviews 2015; 28(3):663-86. doi:10.1128/CMR.00106-14.

6. Sanchez SS. Garcia HH. Nicoletti A. Clinical and magnetic resonance imaging findings of neurotoxocariasis. Frontiers in Neurology 2018; 9:53 doi:10.3389/fneur.2018.00053

7. Sick C, Hennerici MG. Expect the Unexpected: A case of isolated eosinophilic meningitis in toxocariasis. Case Reports in Neurology 2014; 6:259-63. doi: 10.1159/000369060

8. Meliou M, Mavridis LN, Pyrgelis ES, et al. Toxocariasis of the nervous system. Acta Parasitologica 2020; 65:291-299. doi.org/10.2478/s11686-019-00166-1 